\title{
A VERSION OF DISCRETE HAAR TRANSFORM WITH NODES OF $\Pi_{0}$-GRIDS
}

\author{
K.A. KIRILLOV, M.V. NOSKOV
}

\begin{abstract}
We propose a version of the two-dimensional discrete Haar transform with $2^{D}$ nodes forming $\Pi_{0}$-grids associated with the triangular partial sums of Fourier - Haar series of a given function. Due to the structure of $\Pi_{0}$-grids, the computation of coefficients of this discrete transform is based on a cubature formula with $2^{D}$ nodes being exact for Haar polynomials of degree at most $D$, owing to that all the coefficients $A_{m_{1}, m_{2}}^{\left(j_{1}, j_{2}\right)}$ of the constructed transform coincide with the Fourier - Haar coefficients $c_{m_{1}, m_{2}}^{\left(j_{1}, j_{2}\right)}$ for Haar polynomials of degree at most $D-\max \left\{m_{1}, m_{2}\right\} \quad\left(0 \leqslant m_{1}+m_{2} \leqslant d\right.$, where $\left.d \leqslant D\right)$. The standard two-dimensional discrete Haar transform with $2^{D}$ nodes does not possess this property.
\end{abstract}

Keywords: cubature formulae exact for Haar polynomials, discrete Haar transform, $\Pi_{0^{-}}$ grids

\section{INTRODUCTION}

In numerical mathematics, a substantial interest is attracted by the problem on applying cubature formulae exact on some finite system of orthonormalized functions to the discrete Fourier transform w.r.t. this system. For instance, applications of cubature formulae of high trigonometric accuracy to the discrete Fourier transform w.r.t. the trigonometric system were considered in [1].

The idea of employing triangular partial sums of Fourier series for a given function in constructing the discrete Fourier transform, which was realized in paper [1], for the case of the trigonometric system is applied in the present paper for constructing a version of twodimensional discrete Haar transform with nodes forming $\Pi_{0}$-grids being the grids with rather uniformly distributed nodes; if a $\Pi_{0}$-grid is formed by $2^{D}$ nodes, then each of its binary rectangles of area $2^{-D}$ contains exactly one node. Owing to the mentioned structure of $\Pi_{0}$-grids, the calculation of the coefficients for the discrete transform constructed in the present paper is based on a cubature formula with $2^{D}$ nodes being exact for Haar polynomials of degree not exceeding $D$ and due to this fact for each function being a Haar polynomial of degree at most $D-\max \left\{m_{1}, m_{2}\right\} \quad\left(0 \leqslant m_{1}+m_{2} \leqslant d\right.$, where $\left.d \leqslant D\right)$ all the coefficients $A_{m_{1}, m_{2}}^{\left(j_{1}, j_{2}\right)}$ of the constructed transform are equal to the corresponding Fourier-Haar coefficients $c_{m_{1}, m_{2}}^{\left(j_{1}, j_{2}\right)}$. The latter property is absent for the standard discrete Haar transform related with rectangular partial sums of Fourier-Haar series and based on a cubature formula with a rectangular grid of nodes for which the Haar accuracy degree equals $M=\min \left\{M_{1}, M_{2}\right\}$, where $2^{M_{1}}$ and $2^{M_{2}}$ is a number of indices in each component in construction of the nodes of the cubature formula, $M_{1}+M_{2}=D$.

K.A. Kirillov, M.V. Noskov, A version of discrete HaAR transform with NODEs of $\Pi_{0}$-Grids.

(c) Kirillov K.A., Noskov M.V. 2013.

Submitted December 20, 2011. 


\section{MAIN DEFINITIONS AND AUXILIARY STATEMENTS}

In the present paper we employ original definition of the functions $\chi_{m, j}(x)$ introduced by A. Haar [2] and different from that in [3] in the points of discontinuity.

As binary interval $l_{m, j}$ we call the intervals with the end-points $(j-1) / 2^{m-1}, j / 2^{m-1}(m=$ $\left.1,2, \ldots, j=1,2, \ldots, 2^{m-1}\right)$. If the left end-point of a binary segment coincides with 0 , this interval is assumed to be closed on the left; if the right end-point coincides with 1 , the interval is assumed to be closed on the right. Other binary intervals are supposed to be open. The left and the right halves of $l_{m, j}$ (without the center of this binary interval) will be indicated as $l_{m, j}^{-}$ and $l_{m, j}^{+}$, respectively.

As binary rectangles we call the sets $l_{m_{1}, j_{1}} \times l_{m_{2}, j_{2}}$, as closed binary rectangles the closures of these sets $m_{n}=1,2, \ldots, \quad j_{n}=1,2, \ldots, 2^{m_{n}-1}, \quad n=1,2$.

The system of Haar function is constructed by groups; the group no. $m$ is comprised of $2^{m-1}$ functions $\chi_{m, j}(x)$, where $m=1,2, \ldots, \quad j=1,2, \ldots, 2^{m-1}$. We define Haar functions $\chi_{m, j}(x)$ as follows,

$$
\chi_{m, j}(x)= \begin{cases}2^{\frac{m-1}{2}} & \text { as } x \in l_{m, j}^{-}, \\ -2^{\frac{m-1}{2}} & \text { as } x \in l_{m, j}^{+}, \\ 0 & \text { as } x \in[0,1] \backslash \overline{l_{m, j}}, \\ \frac{1}{2}\left[\chi_{m, j}(x-0)+\chi_{m, j}(x+0)\right], & \text { if } x \text { is an interior point of discontinuity }\end{cases}
$$

$\overline{l_{m, j}}=\left[\frac{j-1}{2^{m-1}}, \frac{j}{2^{m-1}}\right], \quad m=1,2, \ldots, j=1,2, \ldots, 2^{m-1}$. In the system of Haar function one also include the function $\chi_{0,1}(x) \equiv 1$ which one relates to the zeroth group.

In the two-dimensional case, as Haar polynomials of degree $d$ we shall call linear combinations with real coefficients of the functions $\chi_{m_{1}, j_{1}}\left(x_{1}\right) \chi_{m_{2}, j_{2}}\left(x_{2}\right)$ referred to as Haar monomials $\left(m_{1}+\right.$ $m_{2}$ is the monomial's degree), $m_{1}+m_{2}=0,1, \ldots, d, j_{n} \in \Lambda_{m_{n}}$,

$$
\Lambda_{m_{n}}= \begin{cases}\left\{1, \ldots, 2^{m_{n}-1}\right\}, & \text { if } m_{n}>0 \\ \{1\}, & \text { if } m_{n}=0\end{cases}
$$

$n=1,2$, at that, at least one of the coefficients at Haar monomials of degree $d$ must be nonzero.

Let a function $f\left(x_{1}, x_{2}\right)$ be defined and summable on $[0,1]^{2}$. We shall say that the cubature formula

$$
I[f]=\int_{0}^{1} \int_{0}^{1} f\left(x_{1}, x_{2}\right) d x_{1} d x_{2} \approx \sum_{i=1}^{N} C_{i} f\left(x_{1}^{(i)}, x_{2}^{(i)}\right)=Q[f]
$$

with nodes $\left(x_{1}^{(i)}, x_{2}^{(i)}\right) \in[0,1]^{2}$ and coefficients at the nodes $C_{i} \in \mathbb{R}(i=1,2, \ldots, N)$ possesses Haar $d$-property, or simply $d$-property, if it is exact for each Haar polynomial $P\left(x_{1}, x_{2}\right)$ of degree at most $d$, i.e.,

$$
Q[P]=I[P] .
$$

Cubature formula (2) will be called a formula of Haar accuracy degree $d$, or $d$-exact, if it possesses $d$-property but not $(d+1)$-property.

The following proposition holds true.

Proposition 1. [4] If cubature formula (2) possesses d-property, the number $N$ of its nodes satisfies the inequality

$$
N \geqslant 2^{d-1}+1
$$

In [3] there employed a definition of Haar functions that is different from the definition introduced in [2], namely, in [3] Haar functions are supposed to be right-continuous at the points of discontinuity, and because of this binary intervals $l_{m, j}\left(m=1,2, \ldots, j=1,2, \ldots, 2^{m-1}\right)$ are 
defined as follows,

$$
l_{m, j}= \begin{cases}{[0,1],} & \text { if } m=1, j=1, \\ {\left[\frac{j-1}{2^{m-1}}, \frac{j}{2^{m-1}}\right),} & \text { if } m \in \mathbb{N} \backslash\{1\}, j \in\left\{1,2, \ldots, 2^{m-1}-1\right\}, \\ {\left[1-\frac{1}{2^{m-1}}, 1\right],} & \text { if } m \in \mathbb{N} \backslash\{1\}, j=2^{m-1} .\end{cases}
$$

We shall say that $2^{d}$ points of unit square $[0,1]^{2}$ form a $\Pi_{0}$-grid if each binary rectangle $l_{m_{1}, j_{1}} \times$ $l_{m_{2}, j_{2}}$ of area $2^{-d}\left(m_{1}+m_{2}=d+2, j_{n}=1,2, \ldots, 2^{m_{n}-1}, n=1,2\right)$ being the Cartesian product of binary segments defined by (3) contains exactly one of these points.

It was shown in [5] that there exist functions $\kappa_{m, j}(x)$ being linear combination of Haar functions from the groups nos. $0,1, \ldots, m$ and satisfying the identity

$$
\kappa_{m, j}(x)= \begin{cases}2^{m} & \text { as } x \in l_{m+1, j} \\ 2^{m-1} & \text { as } x \in \bar{l}_{m+1, j} \backslash l_{m+1, j} \\ 0 & \text { as } x \in[0,1] \backslash \bar{l}_{m+1, j}\end{cases}
$$

$m=0,1,2, \ldots, j=1,2, \ldots, 2^{m}$.

We shall call the functions $\kappa_{m_{1}, j_{1}}\left(x_{1}\right) \kappa_{m_{2}, j_{2}}\left(x_{2}\right) \kappa$-monomials of degree $d$, where $m_{1}+m_{2}=d$, $j_{n}=1,2, \ldots, 2^{m_{n}}, n=1,2$.

The following proposition holds true.

Proposition 2. (4] Cubature formula (2) possesses the d-property if and only if it is exact for all $\kappa$-monomials of degree $d$.

Remark 1. Identity (4) implies that each closed binary rectangle of area $2^{-d}$ is the support of some $\kappa$-monomial of degree $d$, namely, $\overline{l_{m_{1}+1, j_{1}} \times l_{m_{2}+1, j_{2}}}=\operatorname{supp}\left\{\kappa_{m_{1}, j_{1}}\left(x_{1}\right) \kappa_{m_{2}, j_{2}}\left(x_{2}\right)\right\}, m_{n}=$ $0,1,2, \ldots, \quad j_{n}=1,2, \ldots, 2^{m_{n}}, \quad n=1,2$.

Let us prove

Proposition 3. If $K_{d}\left(x_{1}, x_{2}\right)$ is an arbitrary $\kappa$-monomial of degree $d$, then

$$
I\left[K_{d}\right]=\int_{0}^{1} \int_{0}^{1} K_{d}\left(x_{1}, x_{2}\right) d x_{1} d x_{2}=1 .
$$

Proof. Relation (4) yields that $K_{d}\left(x_{1}, x_{2}\right)=2^{d}$ in the interior points of the set $\operatorname{supp}\left\{K_{d}\right\}$. Bearing in mind that $\operatorname{supp}\left\{K_{d}\right\}$ is a binary rectangle of area $2^{-d}$ (Remark 1), we arrive at identity (5). The proof is complete.

The next proposition holds true.

Proposition 4. [4] In the points of continuity of the Haar function $\chi_{m, j}(x)(m=1,2, \ldots$, $j=1, \ldots, 2^{m-1}$ ) the identity

$$
\chi_{m, j}^{2}(x)=\kappa_{m-1, j}(x)
$$

holds true. Everywhere except the points at which the functions $\chi_{k, i}(x)$ and $\chi_{m, j}(x)$ are simultaneously discontinuous (if such points exist), the product of these functions reads as

$$
\chi_{k, i}(x) \chi_{m, j}(x)=\left\{\begin{aligned}
2^{\frac{k-1}{2}} \chi_{m, j}(x), & \text { if }, l_{m, j} \subseteq l_{k, i}^{-} \\
-2^{\frac{k-1}{2}} \chi_{m, j}(x), & \text { if }, l_{m, j} \subseteq l_{k, i}^{+} \\
0 & \text { othwerwise }
\end{aligned}\right.
$$

where $m \geqslant k, i \neq j$ as $m=k$.

Proposition 4 implies

Proposition 5. Everywhere except the points at which Haar polynomial $P\left(x_{1}, x_{2}\right), R\left(x_{1}, x_{2}\right)$ of degree at most $d$ are simultaneously discontinuous (if such points exist), the function $F\left(x_{1}, x_{2}\right)=P\left(x_{1}, x_{2}\right) R\left(x_{1}, x_{2}\right)$ is a Haar polynomial of degree at most $2 d$. 


\section{StANDARD METHOD OF Discrete HAAR TRANSFORM}

Let $f\left(x_{1}, x_{2}\right)$ be a defined and summable on $[0,1]^{2}$ function that can expanded into an absolutely convergent Fourier-Haar series,

$$
f\left(x_{1}, x_{2}\right)=\sum_{n=1}^{2} \sum_{m_{n}=0}^{\infty} \sum_{j_{n} \in \Lambda_{m_{n}}} c_{m_{1}, m_{2}}^{\left(j_{1}, j_{2}\right)} \chi_{m_{1}, j_{1}}\left(x_{1}\right) \chi_{m_{2}, j_{2}}\left(x_{2}\right),
$$

where $\Lambda_{m_{n}}$ is defined by identity (1), $n=1,2$.

Under the standard two-dimensional Haar transform a one-to-one correspondence is made between the sequence of the values of the function $f\left(x_{1}, x_{2}\right)$ at the nodes $\left(x_{1}^{(i)}, x_{2}^{(i)}\right) \in[0,1]^{2}$ $\left(i=1,2, \ldots, 2^{D}\right)$ and the set of the coefficients of this transform $A_{m_{1}, m_{2}}^{\left(j_{1}, j_{2}\right)}\left(m_{n}=0,1, \ldots, M_{n}\right.$, $\left.j_{n} \in \Lambda_{m_{n}}, n=1,2, \quad M_{1}+M_{2}=D\right)$ so that the Haar polynomial

$$
H\left(x_{1}, x_{2}\right)=\sum_{n=1}^{2} \sum_{m_{n}=0}^{M_{n}} \sum_{j_{n} \in \Lambda_{m_{n}}} A_{m_{1}, m_{2}}^{\left(j_{1}, j_{2}\right)} \chi_{m_{1}, j_{1}}\left(x_{1}\right) \chi_{m_{2}, j_{2}}\left(x_{2}\right)
$$

recovers the function $f\left(x_{1}, x_{2}\right)$ in the mentioned nodes,

$$
H\left(x_{1}^{(i)}, x_{2}^{(i)}\right)=f\left(x_{1}^{(i)}, x_{2}^{(i)}\right), i=1,2, \ldots, 2^{D} ;
$$

at that, the number $A_{m_{1}, m_{2}}^{\left(j_{1}, j_{2}\right)}$ is the cubature sum in the cubature formula

$$
\begin{aligned}
& c_{m_{1}, m_{2}}^{\left(j_{1}, j_{2}\right)}=\int_{0}^{1} \int_{0}^{1} f\left(x_{1}, x_{2}\right) \chi_{m_{1}, j_{1}}\left(x_{1}\right) \chi_{m_{2}, j_{2}}\left(x_{2}\right) d x_{1} d x_{2} \approx \\
& \approx 2^{-D} \sum_{i=1}^{2^{D}} f\left(x_{1}^{(i)}, x_{2}^{(i)}\right) \chi_{m_{1}, j_{1}}\left(x_{1}^{(i)}\right) \chi_{m_{2}, j_{2}}\left(x_{2}^{(i)}\right)=A_{m_{1}, m_{2}}^{\left(j_{1}, j_{2}\right)},
\end{aligned}
$$

i.e., an approximate value of the Fourier-Haar coefficient $c_{m_{1}, m_{2}}^{\left(j_{1}, j_{2}\right)}$ for the function $f\left(x_{1}, x_{2}\right)$, $m_{n}=0,1, \ldots, M_{n}, j_{n} \in \Lambda_{m_{n}}, n=1,2$. The nodes $\left(x_{1}^{(i)}, x_{2}^{(i)}\right)\left(i=1,2, \ldots, 2^{D}\right)$, at which the values of the function $f\left(x_{1}, x_{2}\right)$ are calculated, are supposed to belong to the rectangular grid

$$
\left\{\left(\left(2 i_{1}-1\right) 2^{-M_{1}-1},\left(2 i_{2}-1\right) 2^{-M_{2}-1}\right): i_{n}=1,2, \ldots, 2^{M_{n}}, n=1,2\right\} .
$$

Thus, according to (9), the standard method of two-dimensional discrete Haar transform assumes that the set of the indices $m_{1}, m_{2}$ is such that

$$
S\left(x_{1}, x_{2}\right)=\sum_{n=1}^{2} \sum_{m_{n}} \sum_{j_{n} \in \Lambda_{m_{n}}} c_{m_{1}, m_{2}}^{\left(j_{1}, j_{2}\right)} \chi_{m_{1}, j_{1}}\left(x_{1}\right) \chi_{m_{2}, j_{2}}\left(x_{2}\right)
$$

is a rectangular partial sum for series (8) $\left(m_{n}\right.$ in the sum in the right hand side of (11) ranges as $\left.0,1, \ldots, M_{n}, n=1,2\right)$, and calculating of approximate Fourier-Haar coefficients by formula (10) is based on the cubature formula

$$
\begin{gathered}
I[f]=\int_{0}^{1} \int_{0}^{1} f\left(x_{1}, x_{2}\right) d x_{1} d x_{2} \approx \\
\approx 2^{-M_{1}-M_{2}} \sum_{i_{1}=1}^{2^{M_{1}}} \sum_{i_{2}=1}^{2^{M_{2}}} f\left(\left(2 i_{1}-1\right) 2^{-M_{1}-1},\left(2 i_{2}-1\right) 2^{-M_{2}-1}\right)=Q_{1}[f],
\end{gathered}
$$

being the Cartesian product of two cubature formulae with $2^{M_{1}}$ and $2^{M_{2}}$ nodes.

Proposition 6. Haar accuracy degree of cubature formula (12) equals $M=\min \left\{M_{1}, M_{2}\right\}$. 
Proof. Each closed binary rectangle of area $2^{-M}$ contains exactly $2^{\max \left\{M_{1}, M_{2}\right\}}$ nodes of cubature formula (12), and all these nodes are its interior points. Then according to Remark 1 and identities (4), (5), for each $\kappa$-monomial $K_{M}\left(x_{1}, x_{2}\right)$ of degree $M$ we have

$$
Q_{1}\left[K_{M}\right]=2^{-M_{1}-M_{2}} \times 2^{\max \left\{M_{1}, M_{2}\right\}} \times 2^{\min \left\{M_{1}, M_{2}\right\}}=1=I\left[K_{M}\right] .
$$

By Proposition 2 it yields that cubature formula 12 possesses $M$-property. However, it does not possess $(M+1)$-property, since in the case $M=M_{1}$ it is not exact, for instance, for the $\kappa$-monomial $\kappa_{M+1,1}\left(x_{1}\right)$ as well as in the case $M=M_{2}$ for $\kappa_{M+1,1}\left(x_{2}\right)$. Thus, Haar accuracy degree of formula (12) equals $M$. The proof is complete.

Propositions 5, 6 imply that under the condition $m_{1}+m_{2} \leqslant M$ the Fourier-Haar coefficients of the functions $f\left(x_{1}, x_{2}\right)$ being Haar polynomials of degrees at most $M-\max \left\{m_{1}, m_{2}\right\}$ in approximate identity 10$)$ the exact idenity

$$
A_{m_{1}, m_{2}}^{\left(j_{1}, j_{2}\right)}=c_{m_{1}, m_{2}}^{\left(j_{1}, j_{2}\right)}, j_{n} \in \Lambda_{m_{n}}, n=1,2,
$$

holds true, and if $m_{1}+m_{2}>M$, validity of identity 13 can not be guaranteed even for $f\left(x_{1}, x_{2}\right) \equiv$ const for any indices $m_{1}, m_{2}$.

\section{Discrete HaAR transform With nOdes FORMing $\Pi_{0}$-GRids}

The considered version of discrete Haar transform with $2^{D}$ nodes is related with a triangular partial sum of series (8), i.e., with sum (11) for which the subscripts $m_{1}, m_{2}$ appearing in its Fourier-Haar coefficients obey the condition

$$
m_{1}+m_{2} \leqslant d
$$

where $d \leqslant D$ is some fixed natural number. Calculating of the coefficients $A_{m_{1}, m_{2}}^{\left(j_{1}, j_{2}\right)}\left(m_{1}+m_{2} \leqslant d\right.$, $\left.j_{n} \in \Lambda_{m_{n}}, n=1,2\right)$ of this discrete transform is made by formulae (10), at that it is assumed that the nodes $\left(x_{1}^{(i)}, x_{2}^{(i)}\right) \in[0,1]^{2}\left(i=1, \ldots, 2^{D}\right)$ do not lie on the boundaries of the binary rectangles $l_{m_{1}, j_{1}} \times l_{m_{2}, j_{2}}$ of area $2^{-D}\left(m_{1}+m_{2}=D+2\right)$ and form a $\Pi_{0}$-grid. Thus, calculating of the coefficients $A_{m_{1}, m_{2}}^{\left(j_{1}, j_{2}\right)}$ is based on the cubature formula

$$
I[f]=\int_{0}^{1} \int_{0}^{1} f\left(x_{1}, x_{2}\right) d x_{1} d x_{2} \approx 2^{-D} \sum_{i=1}^{2^{D}} f\left(x_{1}^{(i)}, x_{2}^{(i)}\right)=Q_{2}[f]
$$

with the aforementioned location of the nodes.

Proposition 7. The Haar accuracy degree of formula (15) equals D.

Proof. Each closed binary rectangle of area $2^{-D}$ contains exactly one node of cubature formula (15) which is its interior point. Then according to Remark 1 and identities (4), (5), for each $\kappa$-monomial $K_{D}\left(x_{1}, x_{2}\right)$ of degree $D$

$$
Q_{2}\left[K_{D}\right]=1=I\left[K_{D}\right]
$$

By Proposition 2 it implies that cubature formula (15) possesses $D$-property. However, it does not possess $(D+1)$-property, since the number of nodes of each cubature formula possessing $(D+1)$-property is at least $2^{D}+1$ (Proposition 1). Thus, the Haar accuracy degree of formula (15) equals $D$. The proof is complete.

Propositions 5, 7 imply that in the approximative identity (10) one has exact identity (13) for Fourier-Haar coefficients of the functions $f\left(x_{1}, x_{2}\right)$ being Haar polynomials of degree at most $D-\max \left\{m_{1}, m_{2}\right\}$. 
We denote by $\widetilde{N}(d)$ the number of the Fourier-Haar coefficients whose subscripts satisfy inequality (14). It is determined by the formula

$$
\widetilde{N}(d)=2^{d}(0.5 d+1) .
$$

The value of the parameter $d$ in inequality (14) will be fixed as follows,

$$
d=\max \left\{p \in \mathbb{N}: 2^{p}(0.5 p+1) \leqslant 2^{D}\right\} .
$$

Proposition 8. For each $D \in \mathbb{N}$ there exists the unique represention of the form

$$
D=2^{r+1}+r+s-1, \text { where } s=0,1, \ldots, 2^{r+1}, r=0,1,2, \ldots
$$

The value d satisfying identity (17) is determined by the formula

$$
d=2^{r+1}+s-2=D-r-1,
$$

where $r, s$ are values of the parameters in representation (18) of the corresponding number $D$. Proof. For a fixed $r \in \mathbb{N} \cup\{0\}$,

$$
\Delta_{r}=\left\{2^{r+1}+r+s-1: s=0,1, \ldots, 2^{r+1}\right\}
$$

is the set of natural numbers in the segment $\left[2^{r+1}+r-1,2^{r+2}+r-1\right]$, and to different values $s \in\left\{0,1, \ldots, 2^{r+1}\right\}$ different natural numbers in this segment are associated. $\Delta_{r+1}$ is the set of natural number in the segment $\left[2^{r+2}+r, 2^{r+3}+r\right]$ not intersecting with $\left[2^{r+1}+r-1,2^{r+2}+r-1\right]$. Therefore, to different pairs $(r, s)$ there correspond different values $2^{r+1}+r+s-1 \quad(s=$ $\left.0,1, \ldots, 2^{r+1}, \quad r=0,1,2, \ldots\right)$, and representation (18) is thus unique for all values of $D$ for which it exists. And since

$$
\bigcup_{r=0}^{\infty} \Delta_{r}=\bigcup_{r=0}^{\infty}\left\{\left[2^{r+1}+r-1,2^{r+2}+r-1\right] \cap \mathbb{N}\right\}=\mathbb{N},
$$

then it exists for each $D \in \mathbb{N}$.

Let us prove now that the value $d$ determined by formula (19) satisfies condition (17).

Indeed, according to (16),

$$
\widetilde{N}\left(2^{r+1}+s-2\right)=\tilde{N}(D-r-1)=2^{D-1}+2^{D-r-2} s \leqslant 2^{D},
$$

since $s \leqslant 2^{r+1}$. At the same time,

$$
\widetilde{N}\left(2^{r+1}+s-1\right)=\widetilde{N}(D-r)=2^{D}+2^{D-r-1}(s+1)>2^{D} .
$$

The proof is complete.

Remark 2. If value $d$ is determined by formula $(19)$, then $\widetilde{N}(d)=2^{D}$ only in the case $s=2^{r+1}$, i.e., for $D=2^{r+2}+r-1, r=0,1,2, \ldots$. In the case $s=0 \quad\left(D=2^{r+1}+r-1\right.$, $r=0,1,2, \ldots) \tilde{N}(d)=2^{D-1}$, and in the case $0<s<2^{r+1}$ the quantity $\widetilde{N}(d) / 2^{D} \in(0.5,1)$ grows as $s$ grows.

Hence, as $D \neq 2^{r+2}+r-1, \quad r=0,1,2, \ldots$, in triangular partial sum (11) involving Haar monomials $\chi_{m_{1}, j_{1}}\left(x_{1}\right) \chi_{m_{2}, j_{2}}\left(x_{2}\right)$ of degrees at most $d$ there are less terms than in the corresponding rectangular partial sum involving Haar monomials for which $m_{n} \leqslant M_{n}, n=1,2$, $M_{1}+M_{2}=D$. However, bearing in mind the convergence to zero of the coefficients $c_{m_{1}, m_{2}}^{\left(j_{1}, j_{2}\right)}$ as $m_{1}+m_{2}$ increases, one can expect that the quality of the suggested version of discrete Haar transform is not worse than for standard scheme.

At the same time, the suggested in the present paper version of the discrete Haar transform has certain advantages in comparison with the standard transform. Firstly, under the same number of nodes as in the standard transform, the set of the functions $f\left(x_{1}, x_{2}\right)$ satisfying identity 13) is enlarged, and in distinction to the standard transform all the coefficients $A_{m_{1}, m_{2}}^{\left(j_{1}, j_{2}\right)}$ coincide with the corresponding Fourier-Haar coefficients $c_{m_{1}, m_{2}}^{\left(j_{1}, j_{2}\right)}$ of Haar polynomials of several 
first degrees. Secondly, reduction of the number of terms in partial sum (11) leads one to the reduction of calculations amount under the approximation of a function by a mentioned partial

sum, in which instead of Fourier-Haar coefficients $c_{m_{1}, m_{2}}^{\left(j_{1}, j_{2}\right)}$ of a given function one should take the corresponding values $A_{m_{1}, m_{2}}^{\left(j_{1}, j_{2}\right)} \approx c_{m_{1}, m_{2}}^{\left(j_{1}, j_{2}\right)}$.

\section{BIBLIOGRAPHY}

1. V.B. Kashkin, M.V. Noskov, N.N. Osipov. A version of the discrete Fourier transform with nodes on parallelepipedal lattices // Zhurn. vychislit. matem. i matem. fiz. 2001. V. 41, No. 3. P. 355-359. [Comp. math. math. phys. 2001. V. 41, No. 3. P. 329-333.]

2. A. Haar Zur Theorie der orthogonalen Funktionensysteme // Math. Ann. 1910. Vol. 69. P. 331-371.

3. I.M. Sobol'. Multidimensional quadrature formulae and Haar functions. Nauka, Moscow. 1969. (in Russian).

4. M.V. Noskov, K.A. Kirillov Minimal cubature formulas exact for Haar polynomials // J. Approx. Th. 2010. V. 162, No. 3. P. 615-627.

5. K.A. Kirillov, M.V. Noskov. Minimal quadrature formulas accurate for Haar polynomials // Zhurn. vychislit. matem. i matem. fiz. 2002. V. 42, No. 6. P. 791-799. [Comp. math. math. phys. 2002. V. 42, No. 6. P. 758-766.]

Kirill Anatol'evich Kirillov, Siberian Federal University, Kirenskii str., 26, 660074, Krasnoyarsk, Russia E-mail: KKirillov@rambler.ru

Mikhail Valerianovich Noskov, Siberian Federal University, Kirenskii str., 26, 660074, Krasnoyarsk, Russia E-mail: MVNoskov@yandex.ru 